\title{
Le cactus Opuntia tuna (L.) Mill. et ses formes d'usage dans la zone des Niayes au Sénégal
}

\author{
Mame Sokhna Sarr, \\ Centre National de Recherches Forestières, \\ Institut Sénégalais de Recherches Agricoles, Dakar, Sénégal \\ Maimouna Gning, \\ Université Gaston Berger de Saint-Louis, Sénégal \\ Diaminatou Sanogo, \\ Centre National de Recherches Forestières, \\ Institut Sénégalais de Recherches Agricoles, Dakar, Sénégal

\section{Katim Touré, Bienvenu Sambou,} \\ École Nationale Supérieure d'Agriculture \\ (ENSA) de l'Université de Thiès, Sénégal

\section{Mouhamadou Dieye,} \\ Bureau d'Analyses MacroEconomiques, \\ Institut Sénégalais de Recherches Agricoles, Sénégal

\section{Marième Fall Ba, Moussa Dieng, Tamsir Mbaye,} \\ Adja Madjiguene Diallo, \\ Centre National de Recherches Forestières, \\ Institut Sénégalais de Recherches Agricoles, Dakar, Sénégal
}

\section{Doi:10.19044/esj.2020.v16n16p199 URL:http://dx.doi.org/10.19044/esj.2020.v16n16p199}

\section{Résumé}

Opuntia tuna est une espèce bien adaptée dans la zone des Niayes au Sénégal. Sa prolifération assez rapide est due en partie à une sous exploitation suscitant ainsi un envahissement de quelques espaces agropastoraux. Dans une perspective de valorisation et de meilleure gestion de l'espèce, cette présente étude a été conduite pour comprendre les formes d'usages et de gestion de la plante par les populations locales afin de la promouvoir pour renforcer leur résilience. L'étude a porté sur cinq villages de la zone nord des Niayes auprès de petits exploitants de maraichers. Des enquêtes qualitatives par entretiens et groupes de discussion ont été conduites. Elles ont porté sur le niveau de connaissance, les pratiques et usages connus du cactus, les contraintes liées à 
sa présence, les solutions adoptées ou envisagées et le niveau de perception sur sa valorisation. Les résultats ont révélé de nombreux usages de la plante et de ses produits. Plus de la moitié des personnes enquêtées utilise le cactus dans le domaine agricole. L'élevage, la pharmacopée et l'alimentation sont d'autres secteurs également concernés par l'usage du cactus. Les analyses ont montré que la plupart des personnes enquêtées (94\%) admettent un degré d'envahissement très élevé de Opuntia tuna. Le niveau d'envahissement pourrait être liée à la zoochorie selon 38\% des hommes et 33\% des femmes. La bonne adaptation de l'espèce dans la zone serait également un facteur de prolifération selon 33\% des hommes et $23 \%$ des femmes. Afin de pallier la prolifération rapide du cactus les maraichers ont développé des stratégies de gestion telles que le brulis, la coupe et le dessouchage. Une meilleure gestion de cette espèce qui présente de nombreux usages pour les maraichers de la zone des Niayes est nécessaire. De tels usages de l'espèce, valorisés davantage pourrait contribuer de façon significative à la diversification des sources de revenus des communautés locales et au développement durable de la zone des Niayes.

Mots clés : Opuntia tuna, Niayes, Sénégal, usage, valorisation 


\title{
The Cactus Opuntia tuna (L.) Mill and Types of Uses in the Niayes area in Senegal
}

\author{
Mame Sokhna Sarr, \\ Centre National de Recherches Forestières, \\ Institut Sénégalais de Recherches Agricoles, Dakar, Sénégal \\ Maimouna Gning, \\ Université Gaston Berger de Saint-Louis, Sénégal \\ Diaminatou Sanogo, \\ Centre National de Recherches Forestières, \\ Institut Sénégalais de Recherches Agricoles, Dakar, Sénégal

\section{Katim Touré, Bienvenu Sambou,} \\ École Nationale Supérieure d'Agriculture \\ (ENSA) de l'Université de Thiès, Sénégal

\section{Mouhamadou Dieye,} \\ Bureau d'Analyses MacroEconomiques, \\ Institut Sénégalais de Recherches Agricoles, Sénégal

\section{Marième Fall Ba, Moussa Dieng, Tamsir Mbaye,} \\ Adja Madjiguene Diallo, \\ Centre National de Recherches Forestières, \\ Institut Sénégalais de Recherches Agricoles, Dakar, Sénégal
}

\begin{abstract}
In the Niayes region (Senegal), Opuntia tuna is a well-adapted plant. Its rapid propagation is partly due to an underutilization causing an invasion in the area and particularly in the agro pastoral zone. In the perspective to promote the valorization and the best management of the species, this study was conducted to perceive Opuntia tuna local knowledge, type of uses and its management by local population in order to reinforce their resiliencies. Interviews and focus groups were performed in 5 villages in the northern part of the Niayes. The questioners consisted of collecting data about basic knowledge, known usage, constraints and adopted solutions, and eventual form of valorization of the species. Results revealed multiple types of use of the plant. More than $50 \%$ of interviewed use the cactus in the agricultural
\end{abstract}


domain. Other sectors such as livestock farming, pharmacopeia and food nutrition are also concerning. However, 94\% agreed a higher level of invasion of the species. A problem associated with the zoochory according to $30 \%$ of men and $33 \%$ of women. For $33 \%$ of men and $23 \%$ of women, the good adaptation of the species in the area of study is among the main propagation factors. In order to face the rapid proliferation of the cactus, farmers developed some management strategies such as burns, cutting and stump removal. Still, best management strategies of the cactus species in the Niayes areas are needed for the farmers who integrate the plant in their agro pastoral systems in addition to other form of uses in other sectors. The valorization of these multiple usages of cactus may significantly contribute to diversify sources of income of local communities in the Niayes, thereby improving their resilience and sustainable development.

Keywords: Opuntia tuna, Niayes, usage, valorization, Senegal

\section{Introduction}

$\mathrm{Au}$ Sénégal, l'agriculture reste la principale source de revenu des ménages ruraux mais est fortement fragilisée par le changement climatique et les actions anthropiques (ANDS, 2013). Cependant, le niveau d'impact de ces facteurs combinés est souvent tributaire de la vulnérabilité de la localité donnée (First, 2019). En effet, la zone des Niayes, l'une des sept zones agro écologiques du Sénégal qui fournit plus de $2 / 3$ de la production horticole nationale (Ndao, 2012), devient de plus en plus vulnérabilisée. par de nombreuss facteurs dont la dégradation des ressources, la désertification et surtout l'ensablement des cuvettes par les dunes vives qui contraint l'activité des petits exploitants maraichers. La fragilité de cet écosystème agrosylvopastoral très convoité pour l'exploitation de ces ressources naturelles par différents secteurs dont les mines, la pêche et la foresterie, nécessite des innovations technologiques efficaces pour sa préservation.

Une dynamique régressive de la biodiversité végétale a été notée, avec plus de 212 espèces ayant disparu dans la zone entre 1957 et 1992 (Ilboudo, 1992). Cette dégradation de la biodiversité entraîne la dégradation du couvert végétal qui favorise la remobilisation du sable sous l'effet du vent et par conséquent l'ensablement des cuvettes maraichères. Afin de mieux renforcer la protection de ces cuvettes, élargir la gamme des plantes utilisées dans les opérations de reboisement serait une option prometteuse. Certaines espèces comme le cactus (colonisant le paysage des Niayes) pourraient être une alternative d'adaptation au niveau humain et écologique. Le cactus est une plante à fleurs, plus communément appelée Cactaceae, qui est grasse et qui se distingue par leurs types de fleurs et fruits et la présence d'aréoles (Berhaut, 
1975). C'est un groupe d'espèces qui poussent facilement en milieux arides, sablonneux et pauvres, faciles à multiplier et qui peuvent fixer les dunes.

Au Sénégal, les études se focalisent sur l'espèce (ou sous espèce) Opuntia tuna qui est majoritairement rencontrée dans la zone des Niayes (Berhaut, 1975; Hunt, 2006). Cependant, nous n'excluons pas la présence de variétés ou d'autres espèces du genre dans la zone. Ce qui justifie l'usage du terme cactus pour prendre en compte toutes les espèces qui peuvent exister dans la zone. Dans le contexte actuel du changement climatique, le cactus suscite un intérêt environnemental et socio-économique grandissant dans beaucoup de pays comme le Mexique et ceux du Maghreb. En effet, il favorise une diversification de la production agricole, une amélioration de la nutrition humaine et animale, sans compter ses propriétés médicinales avérées (Saenz, 2000 ; Inglese et al.,2002 ; Griffith, 2004). Au regard de ses nombreux atouts, l'exploitation du cactus pourrait offrir des solutions durables à des problèmes environnementaux et socio-économiques cruciaux en milieux arides et semiarides. Au Sénégal, le principal usage du cactus est son utilisation comme haie vive pour protéger les petites parcelles maraichères dans la zone des Niayes. A l'exception de cette technique agroforestière, le cactus reste stéréotypé par son aspect envahissant, laissant en rade son énorme potentiel d'usage multiple dans plusieurs secteurs agroalimentaires et sylvopastoral. C'est dans ce cadre que cette étude a été menée pour mettre en évidence le niveau de connaissances des populations locales, les formes d'utilisation et l'intégration du cactus dans le système agropastoral.

\section{Matériel et méthode}

\subsubsection{Zone d'étude}

L'étude a été menée dans cinq villages de la zone des Niayes au Sénégal. Les Niayes s'étendent sur le littoral nord-ouest entre les régions Dakar et Saint-Louis (figure 1). C'est une bande de $2649 \mathrm{~km}^{2}$, représentant moins de $2 \%$ du territoire national malgré la forte concentration de la population nationale (20\%) (Banque mondiale, 2010). Les sols sont hydromorphes, moyennement organique, de types minéraux bruts, peu évolués (CSE, 2018). Les Niayes sont caractérisées par une très forte densité de la population inégalement répartie avec moins d'habitants dans certains villages que dans d'autres. 


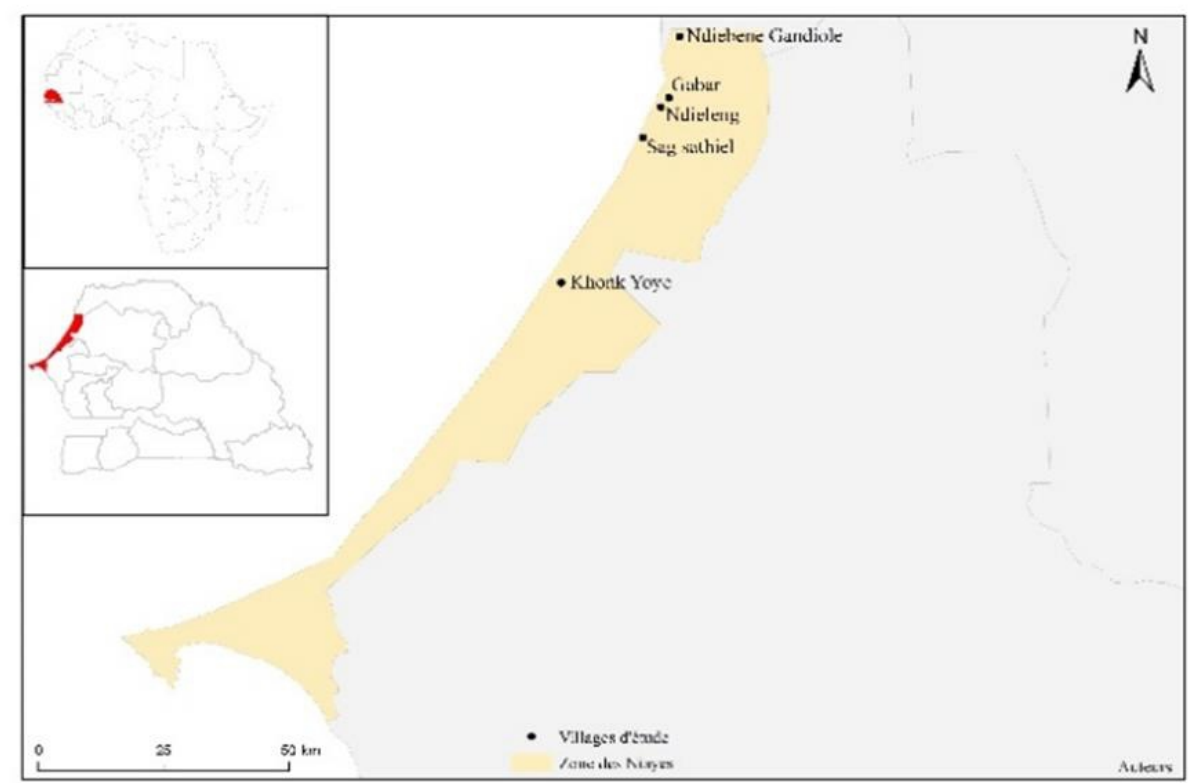

Figure 1 : Carte de localisation des sites d'étude (auteurs)

La zone est essentiellement peuplée par des wolofs, peulhs, maures, diolas et sérères. Les deux premières ethnies sont les plus représentée avec $37 \%$ et $34 \%$ respectivement $(\mathrm{Ndao}, 2012)$. Les peulhs qui sont principalement des éleveurs/pasteurs, se sont progressivement installés à la quête de pâturage saisonnier. Les wolofs quant à eux pratiquent majoritairement le maraichage dans les versants et les bas-fonds.

\subsection{Méthodologie}

Au total cinq (05) villages, Ndieleng, Sag Sathiel, Gabane Peulh, Ndiebene Gandiole et Khonk Yoye, ont constitué les sites de l'étude (figure 1). Ceux-ci ont été choisis en fonction du niveau de prolifération du cactus sur le paysage agraire de la zone des Niayes. Le choix porté sur Opuntia tuna dans cette étude se justifie par sa forte capacité de survie en milieu aride, son intérêt socio-économique et les multiples usages encore méconnus des populations locales.

La méthodologie s'est basée sur des enquêtes qualitatives (entretiens individuels et de groupes) afin d'insister sur les perceptions liées au caractère envahissant et négligé du cactus dans la zone des Niayes. Différentes catégories d'acteurs notamment les maraichers, ont été ciblées pour renseigner les questions portant essentiellement sur le niveau de connaissance du cactus, les pratiques et usages connus du cactus, le degré d'envahissement, les 
contraintes liées à sa présence, les solutions adoptées ou envisagées pour la gestion de l'espèce et le niveau de perception sur sa valorisation.

L'échantillonnage des unités d'observation est non probabiliste (Glaser, 1978). Sur cette base, l'échantillon pour les entretiens individuels est constitué de 66 individus dont 56\% de femmes. Les entretiens de groupes ont été organisés en sélectionnant des participants hommes et femmes d'âges différents. Au total, sept discussions de groupe ont été organisées. Les données collectées ont été analysées suivant une approche socioéconomique descriptive et compréhensive.

\section{Résultats}

\subsection{Caractéristiques socio-économiques des exploitants}

Les personnes enquêtées dans cette étude sont en majorité de petits exploitants maraîchers. Ceux-ci appartiennent à trois groupes ethniques; les wolofs $(55 \%)$ qui sont majoritaires, les peuls $(42 \%)$ et les sérères $(3 \%)$, faiblement représentés (figure 2.a). Toutes les ethnies confondues, les personnes enquêtées sont majoritairement composées d'hommes (56\% de l'échantillon, figure 2.b). Seuls 31\% des personnes enquêtées sont instruits en français. Parmi celles-ci, $8 \%$ ont un niveau primaire, $17 \%$ pour le niveau secondaire et $6 \%$ ont fait des études supérieures. Dans l'échantillon, $27 \%$ des personnes interrogées ont une instruction coranique et $11 \%$ sont alphabétisées en langues nationales (figure 2.c).

Néanmoins, au moment des enquêtes, $52 \%$ des personnes interrogées ne sont affiliées à aucune forme d'organisation (figure 2.d). Les formes d'organisation les plus représentatives sont les Groupements d'Intérêt Economique - GIE - (24\% des personnes interrogées), les Associations Sportives et Culturelles (ASC) regroupant les jeunes des localités (17\%), les plateformes d'innovation $(6 \%)$ et les organisations de producteurs -OP- $(2 \%$ des personnes interviewées). 

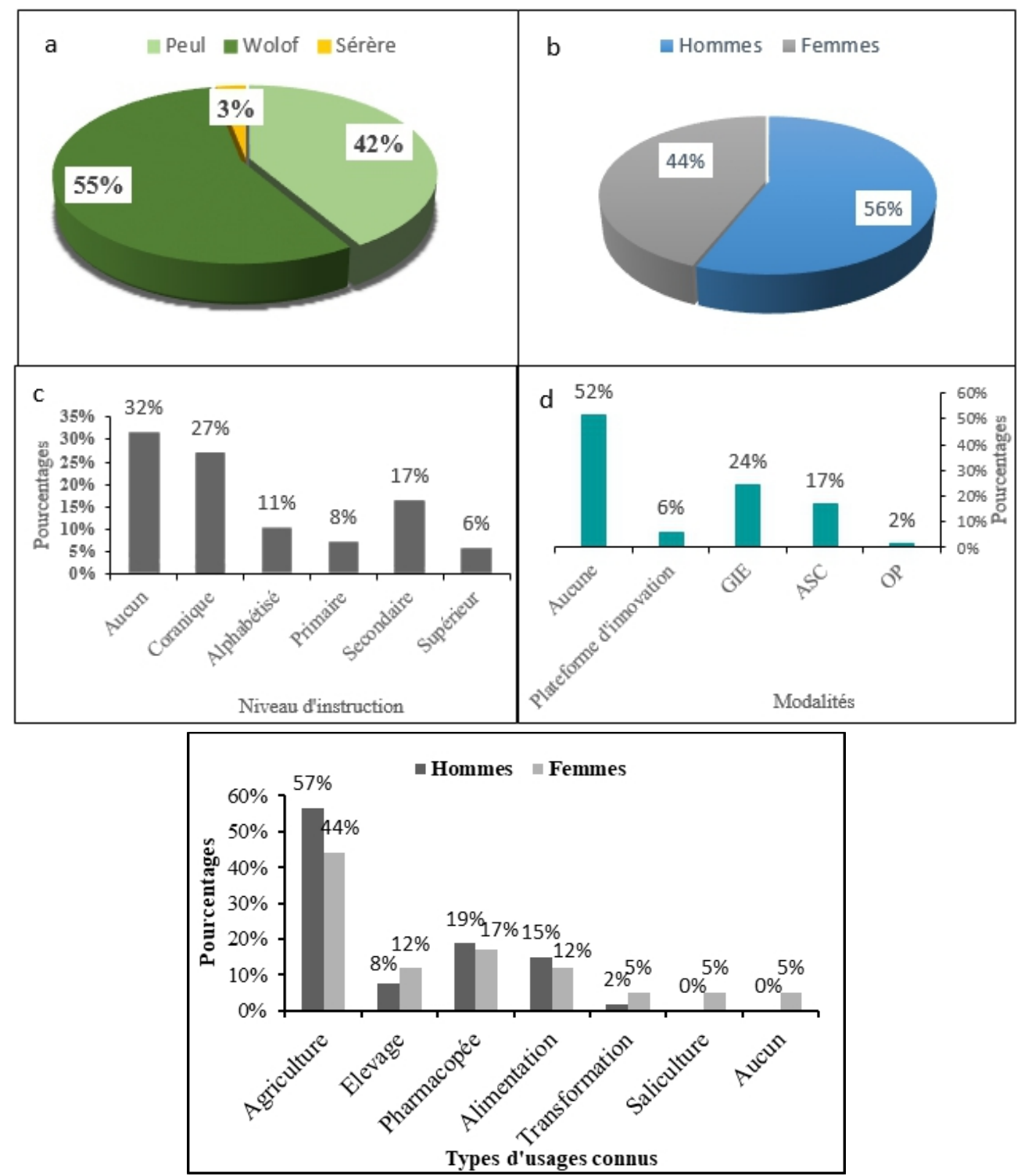

Figure 2 : Représentation des enquêtés selon le capital humain et social

\subsection{Connaissance et usage du cactus}

Les entretiens individuels et collectifs avec la population ont permis de relever le niveau de connaissance du cactus et d'usage par les populations (figure 3). Il s'agit généralement d'usages agricoles, pastorales, pharmaceutiques et alimentaire (nutritionnel). D'autres types d'usage relatifs aux secteurs de la saliculture et de la transformation ont été aussi évoqués. Toutefois, les connaissances inhérentes à ces divers usages sont étroitement liées au sexe. En effet, les usages de nature agricole, pharmaceutique et alimentaire semblent être mieux connus des hommes tandis que ceux de nature 
pastorale, salicole et artisanale sont le plus souvent mentionnés par les femmes (figure 3.a). Il faut aussi noter qu'une proportion très faible (15\% d'hommes et $10 \%$ de femmes) des enquêtés n'ont aucune connaissance des usages faits du cactus.

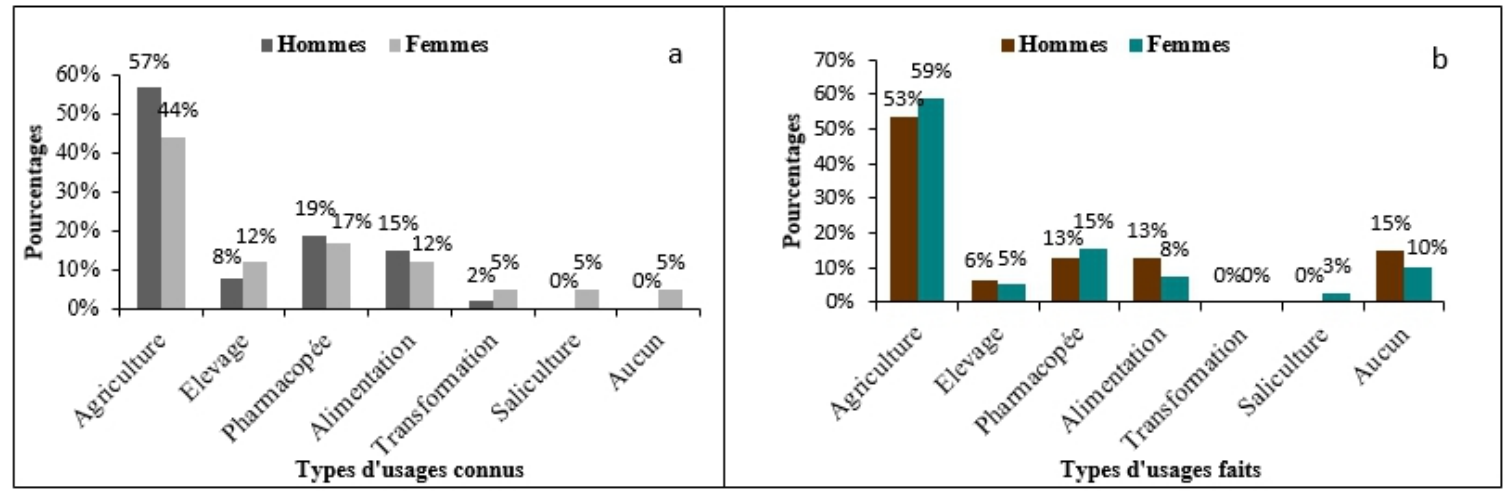

Figure 3 : Perception des usages connus/faits du cactus en fonction du sexe

Ces divers usages connus sur le cactus sont également utilisés par bon nombre de personnes dans leur quotidien. En effet, le cactus est plus fréquemment utilisé dans les secteurs agricole, alimentaire et pharmaceutique aussi bien chez les hommes que chez les femmes. Cette espèce est également utilisée dans une moindre mesure dans l'élevage, la saliculture et la transformation agroalimentaire. En ce qui concerne les usages agricoles et pharmaceutiques, les perceptions féminines l'emportent par rapport à celles des hommes (figure 3.b). Par contre, pour ce qui est des usages pastoraux et alimentaires, les perceptions masculines dominent sur celles des femmes. Les enquêtes ont permis de mettre en évidence les multiples usages du cactus. Il ressort de nos analyses que le cactus sert à la fois de haie vive défensive contre la divagation du bétail dans les cuvettes maraichères et de brise vent pour les cultures maraichères. Les fruits de cette espèce sont également consommés par la population. La sève du cactus est aussi utilisée par les femmes pour le lavage et le traitement des cheveux. En outre, les rides et problèmes de peau, ainsi que les plaies et brulures sont guéris grâce à l'usage de la sève du cactus. Les racines de l'espèce, mélangées avec du sel, sont utilisées par les populations pour le blanchiment des dents mais aussi pour lutter contre la constipation.

Les résultats de nos enquêtes ont montré que les raquettes de cactus sont utilisées pour l'alimentation des chameaux, chèvres et bœufs en période de soudure. Les singes aussi se nourrissent des fruits. Cela pourrait expliquer l'envahissement du cactus dans la réserve de Gueumbeul par l'action des singes qui dispersent ses graines sur le paysage. 


\subsection{Effets du cactus sur le sol}

Les entretiens individuels ont permis de constater des effets du cactus sur le sol. Tandis que certaines personnes perçoivent un effet positif de l'espèce sur le sol en l'occurrence la fertilisation ou encore la fixation des dunes, d'autres notent que la présence de cette espèce dans les cuvettes entraine une dégradation des sols. Les populations qui perçoivent que le cactus n'a aucun effet sur le sol sont majoritaires dans l'échantillon aussi bien chez les hommes que chez les femmes avec respectivement $84 \%$ et $59 \%$ (figure 4).

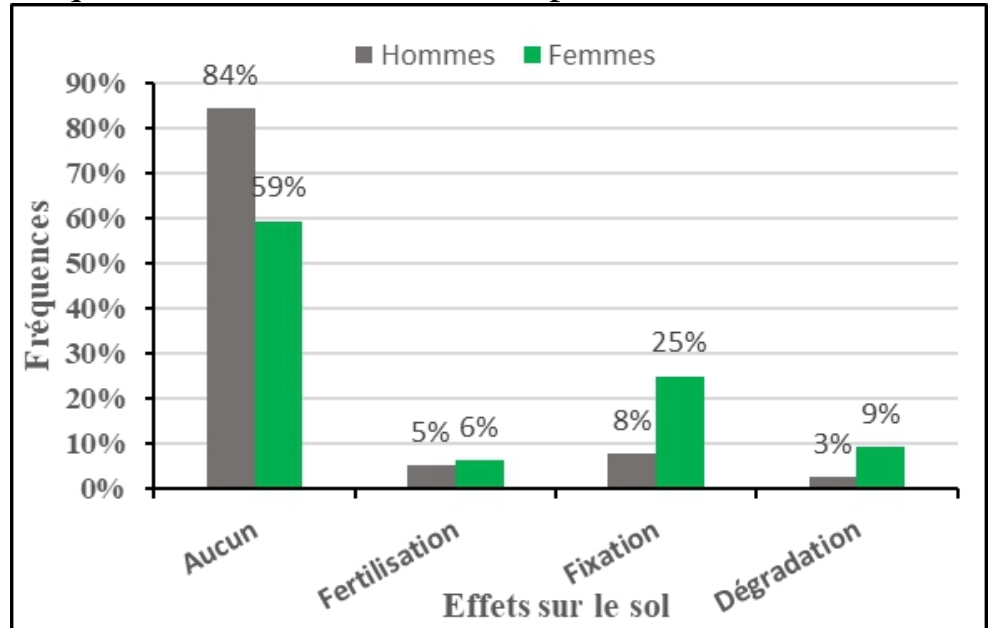

Figure 4 : Perceptions des populations sur les effets du cactus sur le sol

\subsection{Contraintes et stratégies de gestion du cactus}

\subsubsection{Degré d'envahissement et facteurs de prolifération de l'espèce}

La plupart des enquêtés (94\%) mentionnent un degré d'envahissement très élevé du cactus contre une faible proportion qui évoque encore un envahissement assez modéré (6\%) (figure 5.a). Ce constat découle également des entretiens de groupe. Pour les personnes interrogées, le cactus colonise les cuvettes maraichères et une partie importante des espaces de pâturage. Cela a pour conséquence un rétrécissement de l'espace agricole et une diminution des ressources fourragères servant à l'alimentation du bétail. 


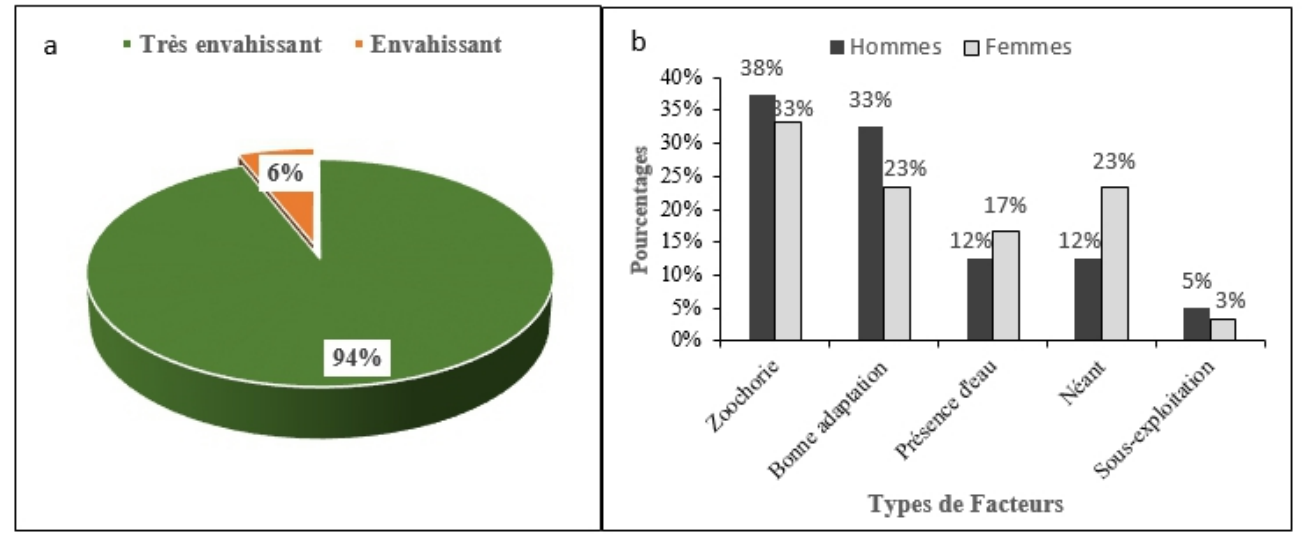

Figure 5 : Perceptions sur le degré d'envahissement et les facteurs de prolifération du cactus

Il ressort des enquêtes individuelles et de groupes un niveau de prolifération du cactus plus rapide que toutes les autres espèces dans le milieu. Trois principaux facteurs de prolifération ont été mis en évidence (figure 5.b).

Le premier facteur est la zoochorie, transport des graines des espèces par les animaux. Il constitue le facteur le plus cité par les hommes et les femmes avec respectivement $38 \%$ et $33 \%$ des enquêtés. Le fruit est consommé par les singes qui transportent ensuite les graines de l'espèce vers d'autres espaces.

Le deuxième facteur est une bonne adaptation de l'espèce. Il est mentionné par plus d'hommes (33\%) que de femmes (23\%). Deux explications ont été avancées par rapport à l'influence de ce facteur sur la prolifération de l'espèce. Il s'agit d'une part de sa capacité de régénération à travers ses cladodes et d'autre part de la présence de conditions pédoclimatiques favorables à son développement. En effet, selon les perceptions collectives, le cactus est une plante réservoir qui conserve beaucoup d'eau dans ses cladodes lesquels contribuent à la germination et à la survie de l'espèce même dans un environnement sec. Les entretiens avec les populations ont permis de mettre en exergue que les conditions pédoclimatiques sont des facteurs qui favorisent un développement rapide de l'espèce étant donné que le cactus est une espèce qui se développe sur sol léger dunaire et avec des températures optimales.

Le troisième facteur est la présence d'eau issue de l'irrigation des cuvettes maraichères. Cette eau est favorable à une prolifération rapide. $\mathrm{Ce}$ facteur a été relevé respectivement par 5\% et 3\% des hommes et des femmes, respectivement lors des entretiens individuels.

\subsubsection{Contraintes liées à l'envahissement et solutions proposées}

Il ressort des enquêtes avec les populations un certain nombre de contraintes liées à la présence de cactus dans les systèmes de production. 
Celles-ci sont la présence des rongeurs et serpents, la présence d'épines sur l'espèce, les contraintes financières liées à sa gestion, l'augmentation des températures qu'il engendre, son caractère envahissant et la dégradation des sols qu'il provoque (figure 6.a). Nos analyses ont montré que pour 33\% des hommes et $32 \%$ des femmes, la présence des rongeurs et serpents constitue une entrave au développement des cultures maraichères dans les Niayes. Les rongeurs causent d'énormes dégâts aux cultures maraichères. Tandis que les reptiles représentent une menace pour l'Homme. Les populations de cactus présentes à proximité des habitations constituent un danger pour les enfants du fait de la présence potentielle de reptiles. Cette analyse a été confirmée par les propos des participants lors des groupes de discussions. La présence d'épines est une limite à la gestion et à la valorisation de l'espèce par la population.

La contribution du cactus à l'appauvrissement des sols de la zone a été également évoqué par les enquêtés qui estiment que ce fait pourrait être lié aux exigences en nutriments de l'espèce. Néanmoins, ce facteur a une incidence faible si on considère sa fréquence de citation. Les perceptions recueillies auprès de la population indiquent aussi que la présence du cactus est responsable des fortes températures observées dans ses zones d'envahissement. La contrainte financière quant à elle tient, selon les enquêtés, au fait que la coupe de l'espèce est très coûteuse. Ce qui serait dû à la présence d'épines, lesquels rendent le cactus difficile à manipuler.

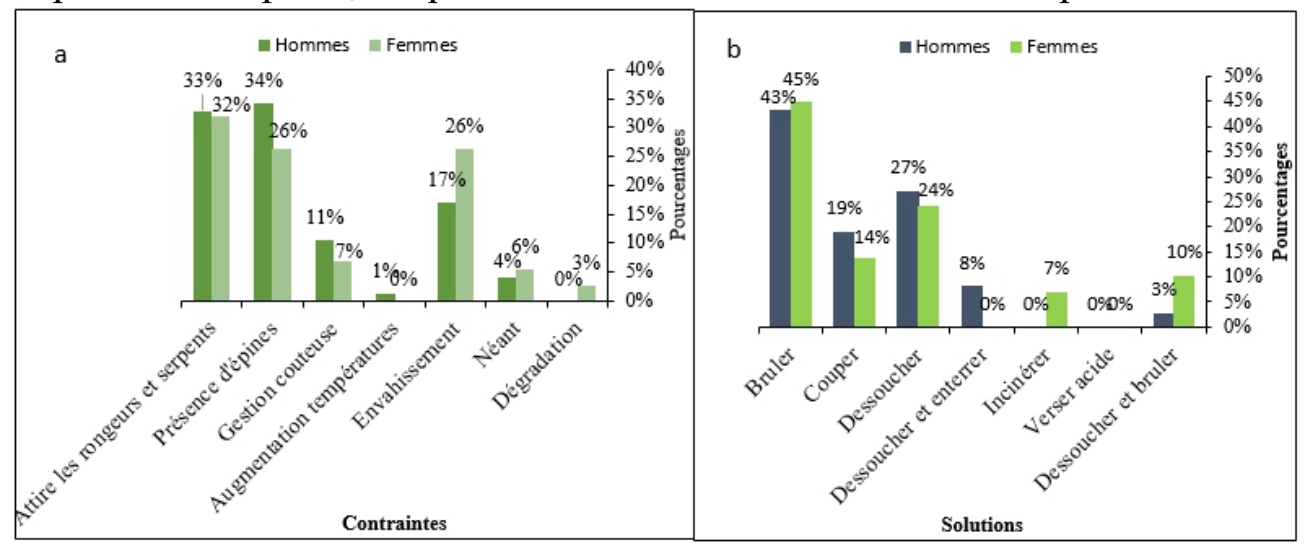

Figure 6 : Perceptions des contraintes de gestion du cactus et solutions apportées

Pour pallier les contraintes et dégâts causés par la présence du cactus, les populations ont développé des stratégies. Celles-ci consistent à couper, brûler, dessoucher, incinérer, ou encore verser de l'acide sur l'espèce pour l'éliminer (figure 6.b). Toutefois, les trois principales solutions utilisées sont le brulis, la coupe (taille) et le dessouchage. Il ressort des enquêtes que $43 \%$ des hommes et $45 \%$ des femmes interrogés optent pour le brulis contre $27 \%$ 
des hommes et $24 \%$ des femmes qui pratiquent le dessouchage. La coupe (taille) est pratiquée par $25 \%$ des hommes et $14 \%$ des femmes.

\section{Discussion}

Contrairement à notre hypothèse d'étude stipulant que le cactus est sous valorisé au Sénégal, diverses formes d'utilisation de la plante et de ses produits dérivés ont été mises en évidence par nos analyses. L'usage dans le domaine agricole est dominant (figure 3). Ce résultat est corroboré par une précédente étude de Diagne (2014) qui avait mis en évidence l'utilisation du cactus comme haie vive défensive dans les Niayes pour la protection des cuvettes maraichères. Notre étude a également révélé que d'autres types d'usage relatifs notamment à la pharmacopée, l'alimentation et l'élevage étaient non seulement connus mais également pratiqués.

Compte tenu des usages multiples du cactus et particulièrement Opuntia ficus indica dans la diversification des sources de revenu, de nombreuses études ont été conduites dans plusieurs domaines (Kumar et al. 2018). Les différentes parties de la plante notamment les raquettes, les fleurs et les fruits sont des produits de haute valeur pouvant constituer une source de revenue pour les populations locales. Les bénéfices nutritionnels des cladodes et des fruits ont été bien documentés (Nefzaoui et Ben Salem, 2001 ; Ben Salem et Abidi, 2007, Misra et al. 2006 ; Souza et al 2009 ; Rocchetti et al. 2018 ; Astello-García et al. 2015). Les fruits consommés sont riches en magnésium, calcium et vitamine $C$. Les cladodes servent de fourrages d'appoint aux ruminants permettant en même temps de réduire les besoins en eau du bétail de plus de $80 \%$. Selon les populations de la zone des Niayes, les fruits servent de nourriture de base aux singes et les enfants l'apprécient beaucoup comme fruit sauvage en plus de son usage pour soigner les plaies. Au cours des groupes de discussions, les agriculteurs et éleveurs ont révélé que leurs troupeaux de bœufs et de chèvres se nourrissaient des cladodes durant les périodes de soudures malgré la présence d'épines. Par conséquent, la promotion du cactus comme fourrage de soudure dans les zones arides vulnérables serait une stratégie très intéressante pour le pastoralisme en milieux arides.

Les propriétés phytothérapeutes soupçonnées par les personnes enquêtées sur les différentes parties de la plante ont déjà fait l'objet d'études sur l'espèce Opuntia tuna en Egypte. Tawfik et al. (2009) ont confirmé la présence de flavonoïde au niveau des fleurs et de mucilage et de betalaine dans les fruits qui ont aussi des propriétés nutraceutiques et cosmétiques. Rochetti et al. (2018) ont aussi révélé la présence de composés phénoliques dans les raquettes du figuier de la barbarie. Ces raquettes ont également des propriétés antioxydantes, anti-virales, anti-inflammatoires et hypoglycémiantes. Ces constats ont été également corroboré par Kumar et al. (2018). L’infusion des 
fleurs séchées est promue pour le traitement du cancer de la prostate et des problèmes urologiques (Inglese et al. 2017). La diversité des usages notée peut susciter un éventuel développement de la chaine de valeur du cactus.

Le rôle joué par le cactus dans la fertilisation ou la dégradation du sol est une observation empirique et un savoir local intéressants. Seule une faible partie des personnes interrogées, 5\% des hommes et $6 \%$ des femmes, est convaincue de ses propriétés fertilisantes sur le sol. Egalement, une faible proportion de la population enquêtée croit à un effet dégradant, alors que la majorité n'ont aucune idée sur l'effet du cactus sur le sol. En effet, les recherches déjà effectuées sur cette question ont révélé que le figuier de la barbarie améliore de façon significative la fertilité des sols dégradés et la richesse spécifique des plantes (Neffar et al. 2011). Ainsi, le cactus aurait un effet positif dans la réhabilitation des terres dégradées et la biodiversité (Neffar et al. (2011). Il serait tout de même intéressant de tester les mêmes effets avec Opuntia tuna, l'espèce la plus répandue dans la zone des Niayes. Le potentiel du cactus sur la fertilisation ou la dégradation du sol constitue un champ d'investigation à adresser et devrait susciter une curiosité scientifique pour la recherche.

L'usage de la plante comme haie vive défensive s'avère contraignante à cause de son développement rapide voire envahissant autour des cuvettes maraichères (figure 2.a). En effet, le manque de gestion appropriée du cactus pousse les populations à le considérer comme une plante envahissante. Une contrainte qu'il tente de pallier avec diverses méthodes dont le brulis, la coupe, le dessouchage et l'incinération (figure 6.b). Cependant, ces pratiques doivent faire l'objet de recherche pour promouvoir les options de gestion les plus durables. Selon $34 \%$ des hommes et $26 \%$ des femmes, la présence d'épine est aussi une contrainte qui rend difficile la gestion de l'espèce. Par ailleurs, de nombreux producteurs maraichers trouvent que la présence d'épines est importante car permet une meilleure protection des parcelles maraichères. L'accompagnement de ces maraichers pour disposer des techniques de gestion efficiente des haies vives de cactus est plus que nécessaire.

\section{Conclusion}

Cette étude a mis en évidence le rôle important que joue le cactus sur le plan socio-économique et environnemental dans les Niayes. En plus d'être une espèce bien adaptée à la zone, le cactus semble être relativement valorisé dans plusieurs domaines. Sa haute valeur environnementale et socioéconomique dans les zones arides suscite un regain d'intérêt pour sa promotion comme stratégie de résilience prometteuse pour les populations locales dans le contexte du changement climatique. Toutefois sa présence engendre des contraintes qui doivent être prises en compte dans une perspective de gestion et de valorisation de la plante et de ses produits et sous- 
produits. La recherche est interpellée dans ce sens pour une meilleure appréhension de son écologie et des stratégies de gestion efficientes au profit des potentiels bénéficiaires au Sénégal.

\section{Remerciements}

Cette étude a été possible grâce à l'appui financier du projet «Renforcement de la gestion et des écosystèmes des Niayes et de la Casamance dans un contexte de changement climatique -République de Sénégal- (PRGTE). Nous remercions Mr Mamadou Sow, agent technique à l'Agence Nationale de Conseil Agricole et Rural (ANCAR) pour son appui lors des enquêtes effectuées sur le terrain.

Conflit d'intérêt : Aucun conflit d'intérêt déclaré.

\section{References :}

1. Agence nationale de la statistique et de la démographie - ANDS. (2013). Recensement général de la population et de l'habitat, de l'agriculture et de l'élevage RGPH

2. Astello-García, M. G., Cervantes, I., Nair, V., del Socorro SantosDíaz, M., Reyes-Agüero, A., Guéraud, F., ... \& de la Rosa, A. P. B. (2015). Chemical composition and phenolic compounds profile of cladodes from Opuntia spp. cultivars with different domestication gradient. Journal of Food Composition and Analysis, 43, 119-130.

3. Banque mondiale (2010). Développement local, Institution et Changement Climatique au Sénégal. Analyse de la situation et recommandations opérationnelles. Rapport Final 89 p.

4. Ben Salem, H., \& Abidi, S. (2007, October). Recent advances on the potential use of Opuntia spp. in livestock feeding. In VI International Congress on Cactus Pear and Cochineal 811, 317-326.

5. Berhaut, J. (1974). Flore illustrée du Sénégal. Tome 2 Clairafrique (édit.), Dakar, Sénégal, 695 p.

6. Centre de Suivi Ecologique - CSE. (2018) Annuaire sur l'environnement et les ressources naturelles du Sénégal. Quatrième édition $388 \mathrm{p}$.

7. Diagne, N. Kh. (2014). Adoption et non adoption de pratiques agroforestières dans la zone de Potou (Louga). Mémoire de fin d'études de master de 1'Université de Cheikh Anta Diop de Dakar (UCAD). Faculté des Sciences Économiques et de Gestion (FASEG). Master Économie Rurale et Politiques Agricoles, $66 p$

8. First, P. J. (2019). Global Warming of 1.5 C An IPCC Special Report on the Impacts of Global Warming of $1.5 \mathrm{C}$ Above Pre-Industrial Levels and Related Global Greenhouse Gas Emission Pathways, in the 
Context of Strengthening the Global Response to the Threat of Climate Change. Sustainable Development, and Efforts to Eradicate Poverty. https://www. ipcc. ch/sr15/. Accessed, 1.

9. Glaser; B. G. (1978). Advances in the methodology of grounded theory: Theoretical sensitivity, Mill Valley, The Sociology Press, 164 p.

10. Griffith, M. P. (2004). The origins of an important cactus crop, Opuntia ficus-indica (Cactaceae): new molecular evidence. American Journal of Botany, 91(11), 1915-1921.

11. Hunt, D. (2006). The new cactus lexicon. Milborne Port, UK, DH books. 900 p.

12. Ilboudo, J.B. (1992). Etat et tendances évolutives de la flore et de la végétation de la Réserve spéciale Botanique de Noflaye (environs de Dakar-SENEGAL), éléments pour un aménagement. Thèse de Doctorat de Troisième Cycle. Dakar : Institut des Sciences de l'Environnement, Université Cheikh Anta Diop, 107 p.

13. Inglese, P., Basile, F., \& Schirra, M. (2002). Cactus pear fruit production in Cacti: Biology and uses, Park S. Nobel, Berkeley, California 163-183

14. Inglese, P., Mondragon, C., Nefzaoui, A., \& Saenz, C. (2017). Crop ecology, cultivation and uses of cactus pear. Food and Agriculture Organization of the United Nations (FAO). 244 p.

15. Kumar, K., Singh, D., \& Singh, R. S. (2018). Cactus Pear: Cultivation and uses. CIAH/Tech./Pub. No73, 38 p.

16. Misra, A. K., Mishra, A. S., Tripathi, M. K., Chaturvedi, O. H., Vaithiyanathan, S., Prasad, R., \& Jakhmola, R. C. (2006). Intake, digestion and microbial protein synthesis in sheep on hay supplemented with prickly pear cactus [Opuntia ficus-indica (L.) Mill.] with or without groundnut meal. Small Ruminant Research, 63(1-2), 125-134.

17. Ndao, M. (2012). Dynamiques et gestion environnementales de 1970 à 2010 des zones humides au Sénégal : étude de l'occupation du sol par télédétection des Niayes avec Djiddah Thiaroye Kao (à Dakar), Mboro (à Thiès et Saint-Louis). Géographie. Université Toulouse le Mirail - Toulouse II. Français. NNT : 2012TOU20018. tel-00718050 $370 \mathrm{p}$.

18. Neffar, S., Beddiar, A., Redjel, N., \& Boulkheloua, J. (2011). Effets de l'âge des plantations de figuier de Barbarie (Opuntia ficus-indica f. inermis) sur les propriétés du sol et la végétation à Tébessa (zone semiaride de l'est algérien). ecologia mediterranea, 37(1), 5-15.

19. Nefzaoui, A., \& Ben Salem, H. (2001). Opuntia: A strategic fodder and efficient tool to combat desertification in the WANA region. Cactus 
(Opuntia species) as Forage. C. Mondragon and S. Perez (eds.), 7390.

20. Rocchetti, G., Pellizzoni, M., Montesano, D., \& Lucini, L. (2018). Italian Opuntia ficus-indica cladodes as rich source of bioactive compounds with health-promoting properties. Foods, 7(2), 24 p.

21. Saenz, C. (2000). Processing technologies: an alternative for cactus pear (Opuntia spp.) fruits and cladodes. Journal of Arid Environments, 46(3), 209-225.

22. Souza, E. J., Guim, A., Batista, Â. M., Santos, K. L., Silva, J. R., Morais, N. A. P., \& Mustafa, A. F. (2009). Effects of soybean hulls inclusion on intake, total tract nutrient utilization and ruminal fermentation of goats fed spineless cactus (Opuntia ficus-indica Mill) based diets. Small ruminant research, 85(1), 63-69.

23. Tawfik, W. A., Abdel-Azim, N. S., Shahat, A. A. A., Hassan, N. M., Ismail, S. I., \& Hammouda, F. M. (2009). Chemical Investigation of Opuntia tuna Mill Growing in Egypt. Australian Journal of Basic and Applied Sciences, 3(1), 96-102. 